\title{
Circuit
}

Musiques contemporaines

\section{La charpente invisible}

Une pratique expérimentale de la phonographie

\section{The Invisible Framework}

\section{Jean Pallandre}

Volume 13, numéro 3, 2003

Électroacoustique : nouvelles utopies

URI : https://id.erudit.org/iderudit/902286ar

DOI : https://doi.org/10.7202/902286ar

Aller au sommaire du numéro

Éditeur(s)

Les Presses de l'Université de Montréal

ISSN

1183-1693 (imprimé)

1488-9692 (numérique)

Découvrir la revue

Citer cet article

Pallandre, J. (2003). La charpente invisible : une pratique expérimentale de la phonographie. Circuit, 13(3), 67-74. https://doi.org/10.7202/902286ar
Résumé de l'article

— Hum... voilà que je commence à comprendre, et voilà comment je te résumerai : tu sembles dire que la phonographie s'est perdue dans la musique, et sans doute au moins à deux titres : au titre du commerce en devenant outil d'enregistrement, cantonnée au simulacre de la reproduction musicale, et au titre des " musiques électroacoustiques », en devenant un timbre nouveau, cantonnée au simulacre de l'appartenance au domaine musical. Or, tu sembles dire que le propre de la phonographie n'est ni là ni ici, que, sans s'opposer en rien à la musique, l'acte phonographique puise ses racines et sa nécessité ailleurs. Dans l'ailleurs de l'image.
Ce document est protégé par la loi sur le droit d'auteur. L'utilisation des services d'Érudit (y compris la reproduction) est assujettie à sa politique d'utilisation que vous pouvez consulter en ligne.

https://apropos.erudit.org/fr/usagers/politique-dutilisation/ 


\title{
La charpente invisible (une pratique expérimentale de la phonographie)
}

\author{
Jean Pallandre
}

Engager sa vie dans une pratique artistique est un long cheminement, une décision de chaque instant et qui à chaque instant doit se déprendre du confort de l'établi, pour inventer sa propre nécessité. Décider de ne savoir jamais, et réclamer avec force le temps et le lieu de cette errance.

Le microphone a été, et demeure, dans le champ des pratiques artistiques, l'outil d'une ouverture incommensurable, en offrant la possibilité, nouvelle, inouie, de créer et de mettre en jeu des images du monde audible. Appelons ce geste "phonographie".

- Je suis phonographiste.

- Ca n'existe pas.

- Non. C'est pour ça.

Parler de "phonographie", en tant que pratique artistique, s'approprier ce terme usuellement réservé à l'industrie pour en faire le paradigme d'une approche expérimentale de l'art sonore lié aux outils de captation et de reproduction du son, c'est décider, pour soi et dans son rapport au monde, d'envisager sa pratique comme le défrichement perpétuel d'un phénomène qui semble d'une portée énorme, et que l'on se refuse à assujettir déjà à quelque mode, à quelque champ, à quelque norme culturelle, à quelque règlement impératif que ce soit.

Expérimental: qui procède de l'expérience.

Expérience : processus dont l'issue est à découvrir / traversée d'un danger.

Le terme « expérimental » désigne une aventure dont on ignore a priori le résultat. 
Je regrette que le terme "enregistrement" soit le nom commun de la phonographie. Enregistrer, n'est-ce pas "placer dans des registres", cataloguer, classer? $\mathrm{Ce}$ terme ne me convient pas, car il passe sous silence, néglige ce phénomène capital : que le microphone dessine une certaine image d'un événement sonore, et que cette image est elle-même encodée sur un certain support selon un procédé qui lui non plus n'est absolument pas neutre. Rien n'est "enregistré ", sinon la trace gravée, l'empreinte d'un acte : l'acte phonographique. On n'enregistre pas le monde, ni même le son du monde. On n'enregistre surtout pas la Musique. On crée des images.

\section{- Ah... fallait le dire plus tôt!}

- Oui, il aurait fallu, on aurait pu, on aurait dû... le dire plus tôt. Aujourd'hui, tout se passe comme s'il fallait revenir en arrière, réenfoncer les portes ouvertes, pour redécouvrir la fécondité artistique d'un acte qu'un siècle de commerce et de culfure ont bien atrophié.

La conception usuelle de l' « enregistrement audio » résume, unifie et simplifie deux opérations qu'il s'agit au contraire de distinguer, si l'on veut y entendre quelque chose : la captation du son, et la gravure. Le microphone est une oreille, une oreille dont on peut jover. En choisissant le micro mis en jeu, en décidant de son placement, de son mouvement, du moment et du style de son entrée en jeu, je mets en scène le monde audible, j'exprime une certaine lecture de ce qui arrive. Car, à vrai dire, ce qui arrive pour moi, phonographiste, n'est plus du tout ce qui arrive pour l'auditeur lambda, écoutant oreille nue. La membrane du micro, s'offrant à moi comme la chance d'un tympan artificiel et mobile, me permet de jouer, d'accentuer, de souligner, de feindre, de masquer, de créer des rapports inouis... Le microphone est un instrument.

Partant de là, la gravure, l'écriture sonore lau sens étymologique: la phonographie), consiste à encoder sur un support la trace de cette expérience. À ce niveau, nous sommes dans une situation d'écriture : une certaine énergie (non pas celle d'un stylographe, d'une imprimante ou d'ondes lumineuses, mais ici l'énergie électrique délivrée par le microphone en jeul produit des signes, encodés selon certaines conventions technologiques, sur un support. Un texte est inscrit. La tâche du phonographiste, c'est d'être à chaque instant conscient du texte qu'il inscrit sur la bande.

Une s'agit donc pas d' "enregistrer», il ne s'agit pas d'emmagasiner, de stocker. $\| n^{\prime} y$ a pas de "son fixé ". II s'agit d'explorer une situation très particulière de relation au réel, où la manifestation audible de ce qui se produit peut être pétrie, sculptée, creusée et transcrite selon l'engagement d'une sensibilité propre. Comprendre cela, sentir cela, permet d'aborder et d'explorer l'acte phonographique en pleine ouverture, et en toute lucidité, sans l'inféoder déjà à quelque objectif extérieur. Nous serons 
sensibles à ce que la membrane des micros nous conte, et orienterons nos choix selon l'accueil que notre sensibilité réservera à ce jeu d'image.

Quelque chose est inscrit sur la bande (ou le disque dur, ou le CDR). Quelque chose qu'il s'agit maintenant de déchiffrer, de lire, de comprendre. Cette opération n'est pas plus anodine que celle qui l'a rendue possible. L'image, il faut lui donner forme, la tailler, l'affirmer. Sans doute l'écriture connaîtra-telle quelques retouches, quelques corrections. Peutêtre sera-telle même profondément modifiée. Tout à ce stade est à nouveau possible, tout est ouvert.

C'est le travail du développement en studio, fondé sur l'écoute, l'expérimentation, l'écoute, l'écoute encore, et l'analyse. II s'agit d'entendre, de dégager, de rendre clair le caractère pertinent de la phonographie en chantier. Le réel sur lequel travaille maintenant le phonographiste, c'est le réel de ce qui est entendu dans le studio. Le temps de son écoute présente n'est plus celui du moment vécu jadis, quand un certain événement avait lieu, en un certain endroit... Le temps de l'écoute présente est un temps propre à l'image phonographique jouée maintenant, avec son début, son déroulement et sa fin. Ce temps, il s'agit de le sculpter, de le forger. C'est une nouvelle phase de développement du projet.

- Développement... c'est un beau terme, ça... dont il est fait usage, tu le remarqueras, en photographie comme en musique. La "phonographie" dont tu parles seraitelle quelque part "entre»?

- Peutêtre... peutêtre la phonographie est-elle la plus belle chance de l'image offerte à la musique, ou le plus beau cadeau du temps fait à l'image... Peutêtre... Et puis, peutêtre tout ce qui arrive, de quelque façon, est-il toujours "entre "...

- Quelque chose de nouveau est inscrit sur la bande. Ce serait comme "le texte d'une image», calibrée, nivelée, sculptée, choisie. Maintenant, cette image est jovée, projetée, présentée dans un espace (studio, salle de concert? salle de cinéma, salon? salle, extérieur ?). Cette présentation est une interprétation, particulière, unique, nécessairement. L'image qui est entendue ici et maintenant ne l'est qu'ici et maintenant. Autre temps, autre lieu, autre instrument de lecture et de diffusion du son, et c'est une autre image qui est entendue, plus ou moins proche, plus ou moins semblable à la première, mais une autre image, une autre réalité.

- Ah... bon... Mais... pourquoi tu fais tout ça?

- Je crois que rien ne m'émeut autant que le présent qui fuit, le réel qui fuit, la vie qui est là, juste là, et pourtant tout ailleurs... La fragilité du verre... La conscience que le 
réel change. On peut croire que la pratique phonographique est une opération technique, qu'on peut diviser en plusieurs phases, plusieurs moments distincts, comme les éléments d'une chaine de construction, mais pas du tout, ce n'est rien de tout ça. C'est seulement une expérience où... le réel s'éprouve... et change... c'est... comment dire, une façon pour moi de me rendre le plus disponible à ce qui est vraiment, à ce que j'entends vraiment, là, maintenant. J'ai employé dix-sept fois le mot "image ", ce n'est donc pas au sens d'un "enregistrement" du réel, mais ce n'est pas non plus au sens de quelque chose d'irréel, ni même au sens d'une copie ou d'une imitation du réel. C'est... "L'image n'est pas une représentation: elle est une empreinte de l'intime et de sa passion'》 (p. 21).

Ouvrir un champ expérimental. Ne pas savoir. S'offrir la possibilité de découvrir. Au départ d'un projet de création, je ne voudrais même pas savoir s'il s'agira de musique, de cinéma, de théâtre ou de pure phonographie. J'aimerais envisager le projet dans la plus grande ouverture, penser qu'il s'agit d'une expérience "phonoculturelle» nouvelle (le terme est d'Alain Savouret). Des conditions, certaines conditions d'expérience sont données. Toujours. Quelque chose est toujours donné : un espace, un contexte, une demande, un enjeu, une échéance, des personnes, un calendrier... Tout cela constitue le cadre d'une expérience. Un protocole expérimental doit être établi (qu'il soit tacite ou rédigé en trois exemplaires importe peu). Ce protocole fixe pour l'ensemble des partenaires concernés les tenants de l'expérience engagée. De quoi s'agit-il ? Qu'est-ce qu'on se propose d'explorer? Dans quelles conditions? Les tenants sont donnés, mais pas les aboutissants. J'ai la nécessité d'ouvrir mes sens à ce qui va se jouer dans la plus grande disponibilité : je n'ai pas d'objectif quant à la teneur artistique du projet. Pas encore. Il faudra du temps, du travail, de l'écoute, du jeu, de la prise de risque, de la recherche, des trouvailles, de l'intuition, du désespoir, du dégoût, de la vanité, de la joie, de l'excitation... À un certain moment, quelque chose se fait jour, qu'il s'agit de voir, de capter, de nommer (au moins pour soil. Cette "chose", si difficile à décrire dans un discours sur la généralité d'une démarche, cette "chose», c'est la charpente, le point d'ancrage sur lequel le sens propre du projet va germer. C'est ce qui fait que ce projet particulier est justement celui-ci et réductible à nul autre. Cette charpente invisible doit être solide et pertinente, car sur elle, l'expérience se développe, une œuvre se bâtit.

À mes yeux, aujourd'hui et en France, tout se passe comme si la création sonore n'avait jamais su exister pour elle-même, défricher ses propres champs, et donc s'était vu colonisée, en particulier par le milieu musical. Comme si la Musique était le seul, unique, exclusif et tyrannique art sonore. Certes, le milieu des arts plastiques accueille de plus en plus de travaux liés au sonore, mais cela reste très marginal, presque anecdotique. Certes, le secteur radiophonique a su créer et préserver un fragile, très fragile espace de création. Mais quels sont les lieux d'expérience, les lieux de diffusion, les lieux de formation, les lieux de pratique, pour une création
1. NANCY, J.-L. (2003), Au fond des images, Paris, Galilée. 
sonore qui ne se revendique ni radiophonique ni musicale? Quels sont les lieux où une expérimentation artistique ouverte du phénomène sonore, en lien avec les outils de captation, traitement et projection du son, soit possible? Comment un artiste qui décide aujourd'hui d'engager son énergie dans l'exploration du sonore, peutil exister, produire, échanger? Les réponses sont qu'il n'y a rien: pas de lieu, pas de réseau, pas de formation, pas de diffusion, pas de reconnaissance. II y a les marges. Seulement les marges. Quelle chance! Le champ est libre, scandaleusement, merveilleusement libre... C'est un problème culturel, politique, mais c'est aussi un problème artistique, ou philologique: car la musique n'est pas le dernier mot de la création sonore.

La musique est l'art du développement, dans un espace et sur une durée, des relations constitutives de la matière sonore. C'est l'expression de la relation intime du son au jeu. Sans s'opposer en rien à cet art, l'acte phonographique puise ses racines et sa nécessité ailleurs. Dans l'ailleurs de l'image. Il faudra développer la réflexion permettant de comprendre en quoi le siège de la phonographie n'est pas celui de la musique. Il faudra répondre à la question que soulevait Luc Ferrari il y a trente ans à propos de ses Presque rien / peu m'importe que mon travail soit ou ne soit pas de la musique, écrivaitil alors, il m'importe plutôt de comprendre ce que $c^{\prime} \operatorname{est}^{2}$ "). II faudra dépasser l'entreprise de légitimation culturelle (" mais oui, bien sûr, il s'agit de musique, et de bonne musique, de la contemporaine, nouvelle, différente... "), tout comme l'exercice de marginalisation ("mais non, bien sûr, ce n'est pas de la musique, c'est un art nouveau, différent, unique... "). I| faudra développer l'investigation sur le sens de cette pratique : la phonographie, et lui inventer les catégories idoines.

- Hum... voilà que je commence à comprendre, et voilà comment je te résumerai : tu sembles dire que la phonographie s'est perdue dans la musique, et sans doute au moins à deux titres: au titre du commerce en devenant outil d'enregistrement, cantonnée au simulacre de la reproduction musicale, et au titre des "musiques électroacoustiques", en devenant un timbre nouveau, limitée à l'appartenance au domaine musical. Or, tu sembles dire que le propre de la phonographie n'est ni là ni ici, et que la profonde richesse de ce jeu mérite bien d'autres chandelles... Nous donnerastu quelques exemples? Quelques perspectives? Quelques visées?

- Je n'ai pas d'exemple à donner, ni surtout aucune leçon. Tu vois, ce que j'ai à dire est très simple: la phonographie est un art merveilleux, dont chacun peut s'emparer. Une pratique artistique quasiment vierge, puisque, comme tu l'as bien compris, ses applications (la création musicale électroacoustique, l'industrie discographique...., pour intéressantes qu'elles soient, dans les champs qui les concernent, n'en épuisent absolument pas la fécondité. Je revendique seulement un espace de liberté et d'exploration pour l'expérience phonographique elle-même. Mais je vois que tu ne te satisfais
2. Je cite de mémoire let on me pardonnera mon imprécision/ ce qui, je crois, était inscrit sur la pochette du disque vinyle Presque rien $n^{\circ} 2$. 
pas de ce vœu généreux... Allons, quelques exemples... quelques désirs réalisés, quelques essais...

Participer à un concert en compagnie de musiciens instrumentistes, improvisateurs. Configurer un dispositif de captation et de restitution du son de tout ou partie du jeu instrumental qui permette, du point d'écoute de l'auditeur, de créer de parfaits "trompe-feuille». Pratiquer. Jover.

Dans un endroit choisi pour sa qualité acoustique et sonore, à un moment donné, réunir deux improvisateurs : I'un phonographiste et l'autre, instrumentiste. Considérant le son du lieu, dans toutes ses manifestations, comme élément à part entière du jeu, improviser. L'instrumentiste développe son jeu dans l'écoute permanente du contexte, le preneur de son invente la phonographie la plus pertinente de la triple relation qui se joue: un lieu, un instrument, des microphones. Pratiquer. Jover.

Participer à un concert en compagnie de musiciens instrumentistes, improvisateurs. Configurer un instrument qui permette la projection d'un grand ensemble de phonographies sur un grand ensemble de haut-parleurs. Explorer dans le mouvement de l'improvisation comment divers niveaux de relations peuvent se jover, se développer, s'associer, se dissocier, entre le jeu des instrumentistes et la projection phonographique. L'instrument que constitue la console de diffusion reliée aux haut-parleurs joue, réagit, participe du moment musical, au même titre que les instruments acoustiques. Mais les espaces sonores suggérés sont pluriels, et complexes. Pratiquer. Jover.

Dans une ville choisie, réunir un photographe, un peintre, un phonographiste... errer... cheminer... capter... saisir... écrire... avec du son, de la lumière, de la peinture... investir les murs de la cité, exposer, partager avec les habitants. Éditer un livre qui n'est ni un roman, ni un disque-objet, ni un catalogue photos, ni un livre de peinture, mais un peu tout cela à la fois: une œuvre photo-phonographique.

Revenir à la simplicité de l'acte solitaire. Entendre ce que les microphones donnent à entendre. Réaliser une phonographie. La jouer. L'éditer.

Dans une ville choisie, deux phonographistes... errer... cheminer... capter... saisir... rencontrer... Chez les habitants, à domicile, dans des moments de relative intimité (les amis, les voisins...), organiser des "veillées haut-parlantes» (le terme est d'Alain 
Savouret), situations conviviales où la phonographie est le support et l'objet d'un échange " phonoculturel ». Observer.

Dans un endroit donné. Un phonographiste, un poète... ou un enfant... Écouter, regarder, sentir... écrire des mots, dire... Réaliser la phonographie d'une parole du lieu dans le lieu. Mettre en scène la relation intime de la voix au paysage.

Capter le silence d'un lieu, et le jouer dans un autre lieu.

- Capter le silence d'un lieu, et le jouer dans un autre lieu. 


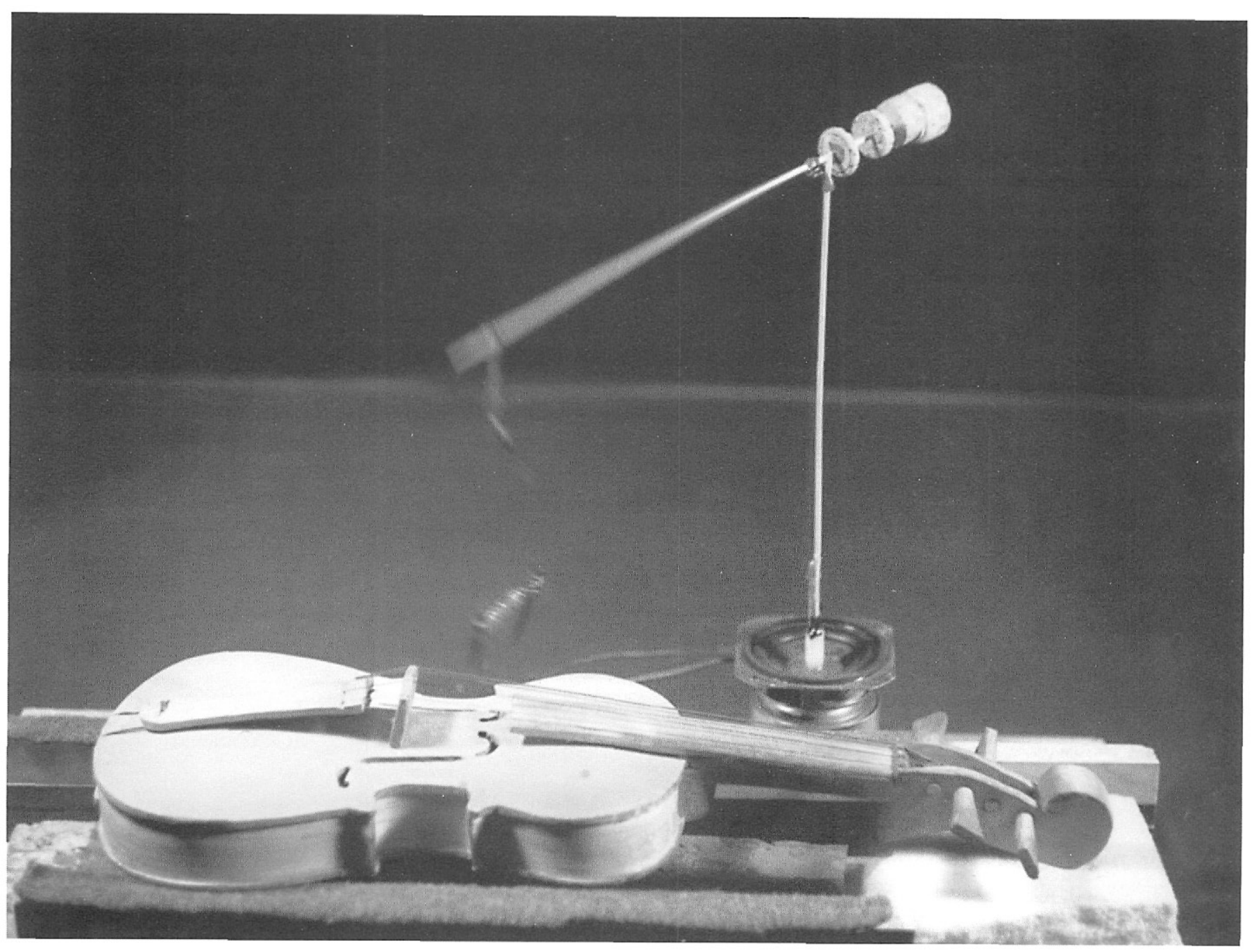

